health systems established COVID-19-only hospitals as the pandemic progressed, our strategy was informed by the ORTP planning tools, which helped to shape the early planning process.

When the first patient was hospitalized at BPH, HCWs were ready to care for that patient and were confident in their training. Having a cadre of nurses, respiratory therapists, and physicians trained in advance to care for patients with COVID-19 was critical in alleviating anxiety, which was expressed by many HCWs throughout the United States. Our model allowed safe care for patients and optimized HCW safety.

The advantages of this model were that PPE use was consolidated, and staff became adept at donning and doffing PPE correctly and identifying best practices to conserve PPE. Concentrating training among a core group of HCWs who then trained others allowed us to quickly train all needed personnel. Having most patients with COVID-19 in one facility allowed patient care protocols to be standardized, bolstered diagnostic and testing capabilities, and facilitated communication among staff.

The main disadvantages of this model of care were that we did not anticipate that patients in Michigan would not be allowed to be transferred to Ohio. This meant that we needed to rapidly duplicate our process in Michigan. Also, transferring critically ill patients at a time when rapid testing was not available meant that patients were transferred with suspected rather than confirmed illness.

Our experience suggests that a hospital dedicated entirely to a novel infection may be a useful strategy for healthcare systems in pandemic planning. This strategy should be considered by large health systems in their advanced planning for future pandemics.
Acknowledgments. We acknowledge all of the administrative and clinical care staff that made planning and patient care possible.

Financial support. No financial support was provided relevant to this article.

Conflicts of interest. Dr.Jennifer Hanrahan was Co-Chair of the Education Committee for the Outbreak Response Training Program. All other authors report no conflicts of interest relevant to this article.

\section{References}

1. Banach D, Johnston B, Al-Zubeid, D, et al. Outbreak response and incident management: SHEA guidance and resources for healthcare epidemiologists in US acute-care hospitals. Infect Control Hosp Epidemiol 2017; 38:1393-1419.

2. SHEA/CDC Outbreak Response Training Program (ORTP). Society for Healthcare Epidemiologists of America (SHEA) website. https://learningce. shea-online.org/content/sheacdc-outbreak-response-training-program-ortp\# group-tabs-node-course-default1/index.php. Accessed November 7, 2020.

3. Outbreak response tool kits. SHEA/CDC Outbreak Response Training Program (ORTP) website. https://ortp.guidelinecentral.com/. Accessed November 7, 2020.

4. Jester BJ, Uyeki TM, Patel A, Koonin L, Jernigan DB. 100 Years of medical countermeasures and pandemic influenza preparedness. Am J Public Health 2018;108:1469-1472.

5. Wang X, Ferro EG, Zhou G, Hashimoto D, Bhatt DL. Association between universal masking in a healthcare system and SARS-CoV-2 positivity among healthcare workers. JAMA 2020;324:703-704.

6. Chen Y, Tong X, Wang J, et al. High SARS-CoV-2 antibody prevalence among healthcare workers exposed to COVID-19 patients. J Infect 2020;81:420-426.

7. Symptoms of coronavirus. Centers for Disease Control and Prevention website. https://www.cdc.gov/coronavirus/2019-ncov/symptoms-testing/ symptoms.html. Updated May 13, 2020. Accessed August 12, 2020.

\title{
Monthly trend in mortality and length of stay among coronavirus disease 2019 (COVID-19) patients: Analysis of a nationwide multihospital US database
}

\author{
Xiaozhou Fan $\mathrm{PhD}^{1}$ (1), Barbara H. Johnson $\mathrm{MBA}^{1}$, Stephen S. Johnston $\mathrm{PhD}^{1}$, Nivesh Elangovanraaj BE ${ }^{2}$, Prerna Kothari \\ BTech $^{2}$, Avrum Spira MD, MSc ${ }^{3}$, Paul Coplan ScD, MBA ${ }^{1}$ and Rahul Khanna PhD, MBA ${ }^{1}$ \\ ${ }^{1}$ Medical Device Epidemiology and Real-World Data Sciences, Johnson \& Johnson, New Brunswick, New Jersey, United States, ${ }^{2}$ MuSigma, Inc, Bangalore, India \\ and ${ }^{3}$ Lung Cancer Initiative, Johnson \& Johnson, New Brunswick, New Jersey, United States
}

Since emerging in the first quarter of 2020, 33.6 million identified cases of coronavirus disease 2019 (COVID-19) caused by severe acute respiratory coronavirus virus 2 (SARS-CoV-2) have been reported, including $>603,000$ associated deaths in the United States as of mid-July, 2021. ${ }^{1}$ Recent evidence suggests that the case fatality rate has been declining among COVID-19 patients, ${ }^{2,3}$ with

\footnotetext{
Author for correspondence: Xiaozhou Fan, E-mail: xfan9@its.jnj.com

Cite this article: Fan X, et al. (2021). Monthly trend in mortality and length of stay among coronavirus disease 2019 (COVID-19) patients: Analysis of a nationwide multihospital US database. Infection Control \& Hospital Epidemiology, 42: 1132-1135, https://doi.org/10.1017/ice.2021.110
}

one study reporting at least a $34 \%$ decline over a 3-month period among all age groups. ${ }^{2}$ However, these studies have been restricted in their generalizability with samples limited to a single state ${ }^{2}$ or health system. ${ }^{3}$

\section{Methods}

Using a nationwide, multihospital database, the Premier Healthcare Database (PHD), ${ }^{4}$ we identified patients with a diagnosis of COVID-19 (ICD-10-CM U07.1) admitted to an inpatient setting (ie, the first admission was considered the index admission). To be included, hospitals were required to provide continuous inpatient data from April 1, 2020 until July 31, 2020, to the PHD. We

(c) The Author(s), 2021. Published by Cambridge University Press on behalf of The Society for Healthcare Epidemiology of America. This is an Open Access article, distributed under the terms of the Creative Commons Attribution licence (http://creativecommons.org/licenses/by/4.0/), which permits unrestricted re-use, distribution, and reproduction in any medium, provided the original work is properly cited. 
Table 1. Patient Characteristics, Treatment, and Outcomes and Provider Characteristics Among COVID-19 Hospitalized Patients in April, May, June, or July

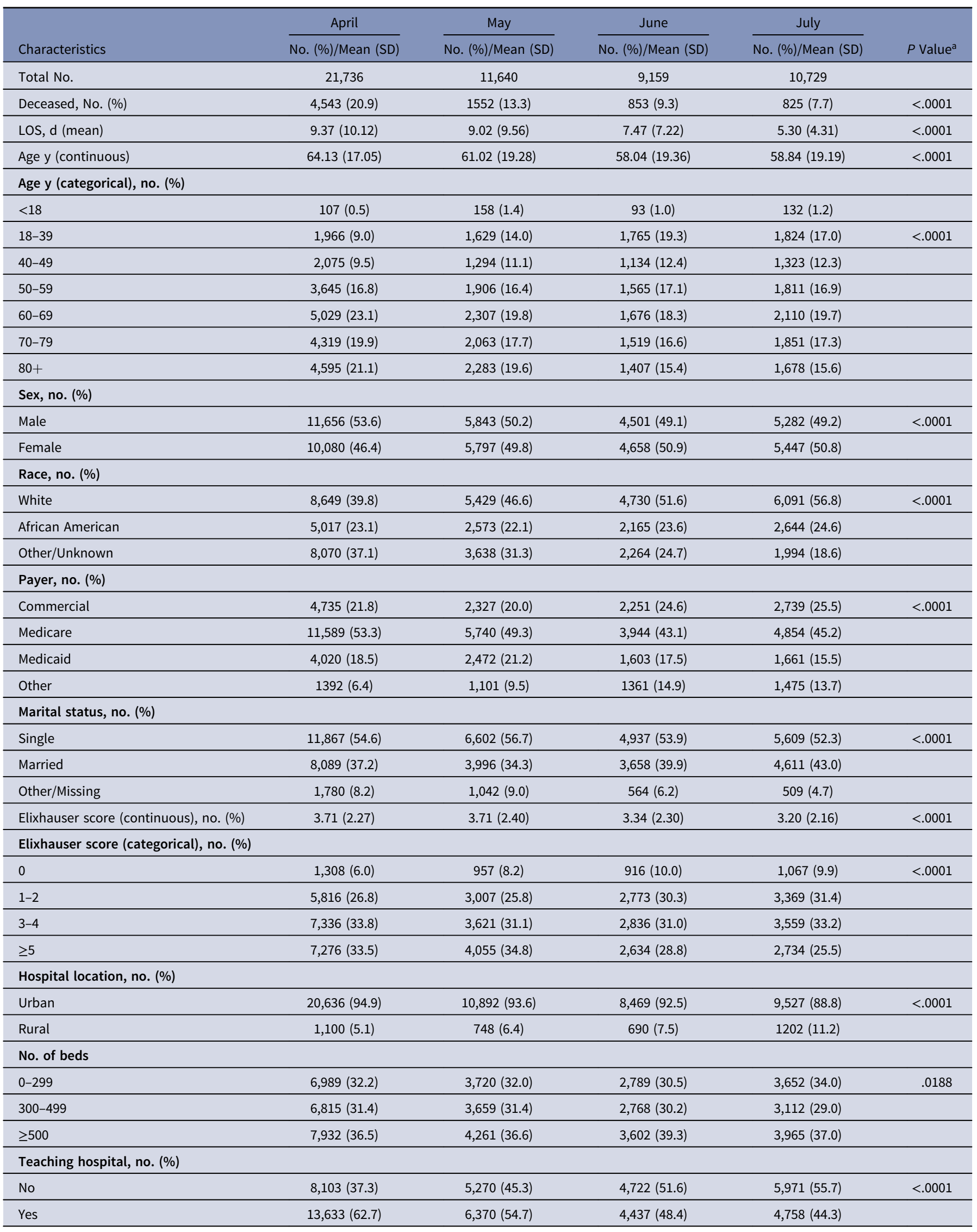


Table 1. (Continued)

\begin{tabular}{|c|c|c|c|c|c|}
\hline & April & May & June & July & \\
\hline \multicolumn{6}{|l|}{ Provider region, no. (\%) } \\
\hline Midwest & $3,845(17.7)$ & $3,410(29.3)$ & $1,794(19.6)$ & $1,761(16.4)$ & \\
\hline West & $515(2.4)$ & $311(2.7)$ & $505(5.5)$ & $611(5.7)$ & \\
\hline Admission to ICU & $5,139(23.6)$ & $2,966(25.5)$ & $2,214(24.2)$ & $2,006(18.7)$ & $<.0001$ \\
\hline Mechanical ventilator & $4,192(19.3)$ & $1,823(15.7)$ & $1,010(11.0)$ & $713(6.6)$ & $<.0001$ \\
\hline Hydroxychloroquine & $11,344(52.2)$ & $1,060(9.1)$ & $102(1.1)$ & $101(0.9)$ & $<.0001$ \\
\hline Azithromycin & $11,253(51.8)$ & $4,631(39.8)$ & $3,809(41.6)$ & $4,479(41.7)$ & $<.0001$ \\
\hline Remdesivir & $79(0.4)$ & $651(5.6)$ & $1,199(13.1)$ & $1,138(10.6)$ & $<.0001$ \\
\hline Methylprednisolone & $5,084(23.4)$ & $2,562(22.0)$ & $1,781(19.4)$ & $1,125(10.5)$ & $<.0001$ \\
\hline Patients admitted to ICU, no. & 5,139 & 2,966 & 2,214 & 2,006 & \\
\hline Deceased, no. (\%) & $2,095(40.8)$ & $976(32.9)$ & $605(27.3)$ & $545(27.2)$ & $<.0001$ \\
\hline LOS, d (mean) & $16.59(14.20)$ & $15.48(12.75)$ & $12.26(9.29)$ & $8.07(5.66)$ & $<.0001$ \\
\hline Patients Aged under 18, no. & 107 & 158 & 93 & 132 & \\
\hline Deceased, no. (\%) & $1(0.9)$ & $0(0.0)$ & $0(0.0)$ & $0(0.0)$ & .1727 \\
\hline LOS, d (mean) & $6.69(9.35)$ & $5.25(7.65)$ & $4.30(4.05)$ & $3.08(2.75)$ & $<.0001$ \\
\hline Patients aged $18-39$ y, no. & 1,966 & 1,629 & 1,765 & 1,824 & \\
\hline Deceased, no. (\%) & $429(11.8)$ & $174(9.1)$ & $103(6.6)$ & $67(3.7)$ & $<.0001$ \\
\hline LOS, d (mean) & $9.94(11.46)$ & $9.68(10.23)$ & $7.78(7.78)$ & $5.35(4.30)$ & $<.0001$ \\
\hline Patients aged 60-69 y, no. & 5,029 & 2,307 & 1676 & 2,110 & \\
\hline Deceased, no. (\%) & $967(19.2)$ & $360(15.6)$ & $176(10.5)$ & $177(8.4)$ & $<.0001$ \\
\hline LOS, d (mean) & $10.58(10.91)$ & $10.39(10.38)$ & $8.81(7.94)$ & $5.84(4.60)$ & $<.0001$ \\
\hline Patients aged $70-79 \mathrm{y}$, no. & 4,319 & 2063 & 1519 & 1851 & \\
\hline Deceased, no. (\%) & $1,289(29.8)$ & $411(19.9)$ & $232(15.3)$ & $222(12.0)$ & $<.0001$ \\
\hline LOS, d (mean) & $9.93(9.76)$ & $10.63(10.03)$ & $8.86(7.77)$ & $6.08(4.78)$ & $<.0001$ \\
\hline Patients aged $\geq 80 \mathrm{y}$, no. & 4595 & 2283 & 1407 & 1678 & \\
\hline Deceased, no. (\%) & $1,671(36.4)$ & $517(22.6)$ & $283(20.1)$ & $303(18.1)$ & $<.0001$ \\
\hline LOS, d (mean) & $8.88(8.63)$ & $8.79(8.35)$ & $8.57(7.26)$ & $6.00(4.49)$ & $<.0001$ \\
\hline
\end{tabular}

Note. COVID-19, the novel coronavirus 2; SD, standard deviation; LOS, length of stay; ICU, Intensive care unit.

${ }^{a} P$ values were based on Pearson's correlation tests for linear trend for continuous, ordinary, and dichotomous variables; and $P$ values were based on $\chi^{2}$ tests for nonordinary categorical variables (eg, race, payer, and marital status).

examined monthly trends (April 2020-July 2020) in mortality and length of stay (LOS) among the hospitalized patients. Then we assessed the relationship between admission month and study outcomes using a generalized estimating equations (GEE) model accounting for potential clustering of outcomes within hospitals, and adjusting for patient characteristics (age, gender, race, marital status, and payer), comorbidity status (Elixhauser comorbidity index score), provider characteristics (region, number of beds, location, and teaching hospital or not), and treatments for COVID-19 (admit to intensive care unit, use of ventilators, 
hydroxychloroquine, azithromycin, remdesivir, convalescent plasma, anticoagulants, dexamethasone, and methylprednisolone). Furthermore, we conducted a stratified GEE analysis according to age. Analyses were performed using $\mathrm{R}$ version 4.0.0 software ( $\mathrm{R}$ Foundation for Statistical Computing, Vienna, Austria).

\section{Results}

The final sample included 53,264 COVID-19 patients from 302 hospitals in 4 geographic regions: 118 (39.1\%) were admitted in the South, $104(34.4 \%)$ in the Midwest, $62(20.5 \%)$ in the Northeast, and $18(6.0 \%)$ in the West. Furthermore, 21,736 were admitted in April; 11,640 were admitted in May; 9,159 were admitted in June; and 10,729 were admitted in July. The distributions of mortality rate, LOS, and covariates included in the GEE model by admission months in the overall study cohort, in patients admitted to ICU, and in each age group are shown in Table 1 . The mean age of the patients decreased from 64.1 years $( \pm 17.1)$ in April to 58.8 years $( \pm 19.2)$ in July $(P$ trend $<.0001)$. The mean Elixhauser comorbidity index score,${ }^{5}$ used to asseses comorbidities among study patients, decreased from $3.7( \pm 2.3)$ in April to $3.2( \pm 2.2)$ in July $(P$ trend $<.0001)$, and the proportion of patients with an index score of 5 and above (indicating high underlying comorbidity burden) fell from $33.5 \%$ in April to $25.5 \%$ in July ( $P$ trend $<.0001)$. The proportion of patients on mechanical ventilation decreased from $19.3 \%$ in April to $6.6 \%$ in July $(P$ trend $<.0001)$.

The mortality rate among hospitalized COVID-19 patients declined from $20.9 \%$ in April to $7.7 \%$ in July ( $P$ trend $<.0001)$. The LOS also declined during this period, from 9.4 days $( \pm 10.1)$ in April to 5.3 days $( \pm 4.3)$ in July $(P$ trend $<.0001)$. Results from GEE analysis after accounting for the differences in the patient population by admission months indicate that COVID-19 patients admitted in May had $43 \%$ lower odds of mortality (odds ratio [OR], 0.57; 95\% confidence interval [CI], 0.52-0.62), whereas those admitted in June had 56\% lower odds of mortality (OR, 0.44; 95\% CI, 0.39-0.49), and those admitted in July had 57\% lower odds of mortality (OR, 0.43; 95\% CI, 0.39-0.49) compared to patients admitted in April. Compared to the LOS among COVID-19 patients admitted in April, patients admitted in May had an average $4 \%$ shorter LOS (adjusted ratio of means, 0.96; 95\% CI, 0.940.98 ), whereas patients admitted in June and July had $26 \%$ (adjusted ratio of means, $0.74 ; 95 \% \mathrm{CI}, 0.72-0.76$ ) and $46 \%$ (adjusted ratio of means, 0.54; 95\% CI, 0.53-0.56) shorter LOS, respectively. The age-stratified GEE analysis revealed that the decrease in mortality was more pronounced in COVID-19 patients aged 60 years or older, and the decrease after June 2020 in LOS was consistent across all age groups. Among patients admitted to an intensive care unit (ICU), the mortality rates were $40.8 \%$ in April, $32.9 \%$ in May, $27.3 \%$ in June, and $27.2 \%$ in July $(P$ trend $<.0001)$. The LOSs for COVID-19 patients admitted to ICU were 16.6 days $( \pm 14.2)$ in April, 15.5 days $( \pm 12.8)$ in May, 12.3 days $( \pm 9.3)$ in June, and 8.1 days $( \pm 5.7)$ in July $(P$ trend $<.0001)$.

\section{Discussion}

In the initial stages of the pandemic in the United States, the patients hospitalized with COVID-19 were mostly older individuals with high comorbidity burden, ${ }^{6}$ but as the pandemic has spread, an increasing number of hospitalizations have been reported among younger individuals. ${ }^{7}$ The decrease in average age and comorbidity status among COVID-19 cases as the pandemic has progressed has also been reflected in our sample of hospitalized patients. Reductions in mortality, as seen in this study, mirror those from other recent studies. ${ }^{2,3}$ We also observed significant reductions in LOS, especially in June and July (vs April); together with mortality reductions, these reductions suggest that the evolving clinical care for hospitalized COVID-19 patients is producing incremental gains in outcomes. The improvements in mortality and LOS were notable for COVID-19 patients admitted to ICUs, reflecting the incremental treatment gains among more severe cases. Several different medical and public health policy factors may have contributed to these meaningful declines in mortality and LOS: improved clinical experience among physicians, better hospital protocols in patient management, more effective treatment options, increased usage of masks and social distancing measures reducing the volume of hospitalization pressure on healthcare systems. ${ }^{8}$

Acknowledgments. We extend our appreciation to Rusha Chaudhuri, Harikumaran R. Dwarakanathan, Aatheep Gurubaran, Joseph Guilfoyle, and Pragya Rai for their assistance with this study.

Financial support. This study was sponsored by Johnson \& Johnson.

Conflicts of interest. All authors report no conflicts of interest relevant to this article.

\section{References}

1. Coronavirus disease 2019 (COVID-19): cases in US Centers for Disease Control and Prevention website. https://www.cdc.gov/coronavirus/2019-ncov/casesupdates/cases-in-us.html. Published 2020. Accessed March 30, 2021.

2. Harris JE. COVID-19 case mortality rates continue to decline in Florida. medRxiv 2020. doi: 10.1101/2020.08.03.20167338.

3. Horwitz L, Jones SA, Cerfolio RJ, et al. Trends in COVID-19 risk-adjusted mortality rates in a single health system. medRxiv 2020. doi: 10.1101/2020. 08.11.20172775.

4. Premier Healthcare Database White Paper: data that informs and performs, March 2, 2020. Premier Applied Sciences website. https://learn.premierinc. com/white-papers/premier-healthcaredatabase-whitepaper. Published March 2020. Accessed March 30, 2021.

5. Elixhauser A, Steiner C, Harris DR, Coffey RM. Comorbidity measures for use with administrative data. Med Care 1998;36:8-27.

6. Garg S, Kim L, Whitaker M, et al. Hospitalization rates and characteristics of patients hospitalized with laboratory-confirmed coronavirus disease 2019-COVID-NET, 14 states, March 1-30, 2020. Morb Mortal Wkly Rep 2020;69:458-464.

7. Greene DN, Jackson ML, Hillyard DR, Delgado JC, Schmidt RL. Decreasing median age of COVID-19 cases in the United States: changing epidemiology or changing surveillance? PLoS One 2020;15(10):e0240783.

8. VoPham T, Weaver MD, Hart JE, Ton M, White E, Newcomb PA. Effect of social distancing on COVID-19 incidence and mortality in the US. medRxiv 2020. doi: $10.1101 / 2020.06 .10 .20127589$. 